\title{
Mass dependence of pseudocritical temperature in mean field approximation
}

\author{
Zhan Bai, ${ }^{1,}$ Lei Chang, ${ }^{2, *}$ Jingyi Chao $\odot,{ }^{3, *}$ Fei Gao, ${ }^{4}$ and Yu-xin Liu ${ }^{1,5,6, \dagger}$ \\ ${ }^{1}$ Department of Physics and State Key Laboratory of Nuclear Physics and Technology, \\ Peking University, Beijing 100871, China \\ ${ }^{2}$ School of Physics, Nankai University, Tianjin 300071, China \\ ${ }^{3}$ Institute of Modern Physics, Chinese Academy of Sciences, Lanzhou 730000, China \\ ${ }^{4}$ Institut für Theoretische Physik, Universität Heidelberg, \\ Philosophenweg 16, 69120 Heidelberg, Germany \\ ${ }^{5}$ Collaborative Innovation Center of Quantum Matter, Beijing 100871, China \\ ${ }^{6}$ Center for High Energy Physics, Peking University, Beijing 100871, China
}

(Received 29 July 2020; revised 4 June 2021; accepted 8 June 2021; published 7 July 2021)

\begin{abstract}
We implement the Dyson-Schwinger equation approach to study the mass dependence of pseudocritical temperature of QCD crossover at zero chemical potential. For the quark-gluon vertex, we take simply the rainbow approximation, while for the gluon propagator, we make use of various dressing functions. We analyze the scaling behavior with these different gluon models and obtain the critical exponent consistent with the mean field value for $3 \mathrm{D} \mathrm{O}(4)$ universality class, which means the rainbow truncation will lead to the mean field result, regardless of the gluon dressing model. The size of critical region is up to $m_{0} \leq$ 1-2 MeV in this mean field approximation, which sets naturally an upper bound of the critical region since the fluctuations beyond mean field usually diminish the critical region. The pseudocritical temperature corresponds to the maximum value of the chiral susceptibility, and for the chiral susceptibility of a certain percentage of its maximum value, the corresponding temperature might be mass independent. We analyze this percentage and studied the pion mass range in which the corresponding temperature remain constant. The results show that this percentage and the pion mass range depend on the details of interaction kernel, which differs in gluon models.
\end{abstract}

DOI: 10.1103/PhysRevD.104.014005

\section{INTRODUCTION}

The basic degrees of freedom in QCD_gluons and quarks - do not exist as asymptotic states; i.e., they are confined inside hadrons and cannot be detected directly at low temperature and low chemical potential. The interaction responsible for this phenomenon also appears to generate more than $98 \%$ of the mass of visible matter [1-3]. These features are known as confinement and dynamical chiral symmetry breaking (DCSB). As the temperature ( $T$ )

\footnotetext{
* Corresponding author. leichang@nankai.edu.cn

Corresponding author. yxliu@pku.edu.cn

Fresent address: College of Physics and Commun. Electronics; Jiangxi Normal University, Nanchang 330022, China.

baizhan@pku.edu.cn

Published by the American Physical Society under the terms of the Creative Commons Attribution 4.0 International license. Further distribution of this work must maintain attribution to the author(s) and the published article's title, journal citation, and DOI. Funded by SCOAP.
}

and chemical potential $(\mu)$ increase, owing to the asymptotic freedom of QCD, QCD matter transits from a DCSB phase to a dynamical chiral symmetry preserving (DCS) phase [1,4-15]. Such features cover a vast array of empirical aspects, from gluon and quark interactions at the highest energies achievable with the heavy ion collision, to the nature of nuclear matter in the inner part of compact stars, which then deliver a sketch of QCD phases at finite temperature and chemical potential (see, e.g., Refs. [4,7,12-23]).

It has been shown that for massless up and down quarks, the transition from the DCSB phase to the DCS phase at zero chemical potential and high temperature is a secondorder phase transition, and the singular behavior of this phase transition can be described by some critical exponents only related to the dimension and symmetry of the theory. This is the hypothesis of universality (see, e.g., Ref. [24]). The chiral phase transition belongs to the threedimensional (3D) $\mathrm{O}(4)$ universality class, if the $U_{A}(1)$ anomaly still exists in the phase transition region $[11,25-$ 29] as well as in the chiral limit [30-33]. If $U_{A}(1)$ symmetry has been restored sufficiently, it is expected to 
be a larger 3D universality class. Recent calculation confirms that the temperature for the $U_{A}(1)$ symmetry to be restored may in fact be much higher than the pseudocritical temperature of the chiral crossover (see, e.g., Ref. [34-38]). If the quarks take their physical mass, the second-order phase transition at zero chemical potential will soften to a crossover, but there will be a critical end point (CEP) somewhere on the $T-\mu$ plane, and the CEP is also expected to be in the 3D Ising class $[28,39]$. To determine the location of the CEP (and/or the tricritical point) on the QCD phase diagram with nonperturbative QCD approaches, one should in principle carry out the calculations on the whole $T-\mu$ plane with the appropriate criterion, for example, the divergence of the chiral susceptibility (e.g., Refs. [7,40]). However, the lattice QCD calculation, which is commonly believed as a fundamental nonperturbative QCD approach, is not available for the finite chemical potential case in principle, due to the sign problem. Then, based on the critical behavior analysis, the phase structure at vanishing density could be helpful for the extrapolation to finite density so as to determine the CEP at physical quark mass. For example, lattice QCD calculations at small chemical potential which rely on the Taylor expansions at zero chemical potential [41-44] have shown that the phase transition temperature at the chiral limit could be considered as the upper bound for the temperature of the CEP [45-48].

However, the reconstruction with a certain universality class is quite subtle, owing to the uncertainty of the size of the critical region. Generally, the fluctuations drive deviation of the scaling behavior, and a detailed analysis reveals that the actual size of the scaling behavior could be only up to $m_{\pi} \sim 1 \mathrm{MeV}$ [45].

Since the Dyson-Schwinger equations (DSEs) approach has been shown to be a sophisticated QCD approach $[12,23,49-54]$, we study the scaling behavior of the chiral susceptibility in the DSEs approach in this paper. In order to have a clearer signal of critical behavior and broader critical region, we focus on our computations in rainbow approximation, which is generally believed to be a mean field approximation [30-32]. As for the gluon model, we adopt the Qin-Chang (QC) model [55], which has a finite value in the deep infrared and is different from the ones used in previous mean field calculations [30-32]. In order to better explore the critical behavior and the dependence of the pseudocritical temperature on the current quark mass, we will take parameters in the model to extreme values. In these cases, the QC model will become the MunczekNemirovsky (MN) model [56] and the contact model (see, e.g., Refs. [57-60]). Also, for all the gluon model we use, a temperature-dependent damping factor will be introduced and adjusted to fit the recent calculated results in the functional renormalization group (FRG) approach of QCD [29]. Then, we will compare our results with the FRG result [29] and lattice QCD result [11].
The remainder of this paper is organized as follows. In Sec. II, we reiterate briefly the DSEs approach at finite temperature including the models and the chiral phase transition criterion. Section III represents our results and discussions. Finally, we summarize in Sec. IV.

\section{QUARK GAP EQUATION AT FINITE TEMPERATURE}

The quark propagator $S(p)$ is essential to the study of chiral phase transition. At finite temperature, according to the Lorentz structure analysis, the inverse of the quark propagator can be decomposed approximately as

$$
\begin{aligned}
S\left(\vec{p}, \omega_{n}\right)^{-1}= & i \vec{\gamma} \cdot \vec{p} A\left(\vec{p}^{2}, \omega_{n}^{2}\right) \\
& +i \gamma_{4} \omega_{n} C\left(\vec{p}^{2}, \omega_{n}^{2}\right)+B\left(\vec{p}^{2}, \omega_{n}^{2}\right),
\end{aligned}
$$

where $A\left(\vec{p}^{2}, \omega_{n}^{2}\right), B\left(\vec{p}^{2}, \omega_{n}^{2}\right)$ and $C\left(\vec{p}^{2}, \omega_{n}^{2}\right)$ are scalar functions and $\omega_{n}=(2 n+1) \pi T$ is the Matsubara frequency for quarks. Notice that there should in principle be another term proportional to $\sigma_{\mu \nu}$ in the decomposition of quark propagator. However, its contribution to order parameter is small $[23,61]$ and therefore is omitted here in order to ease the computation task.

The quark propagator at finite temperature can be determined by the gap equation

$S\left(\vec{p}, \omega_{n}\right)^{-1}=Z_{2}\left(i \vec{\gamma} \cdot \vec{p}+i \gamma_{4} \omega_{n}\right)+Z_{4} m_{0}+Z_{2}^{2} \Sigma\left(\vec{p}, \omega_{n}\right)$,

$$
\begin{aligned}
\Sigma\left(\vec{p}, \omega_{n}\right)= & T \sum_{l=-\infty}^{\infty} \int \frac{d^{3} q}{(2 \pi)^{3}} g^{2} D_{\mu \nu}\left(\vec{p}-\vec{q}, \Omega_{n l} ; T, \mu\right) \\
& \times \frac{\lambda^{a}}{2} \gamma_{\mu} S\left(\vec{q}, \omega_{l}\right) \frac{\lambda^{a}}{2} \Gamma_{\nu}\left(\vec{q}, \omega_{l}, \vec{p}, \omega_{n}\right),
\end{aligned}
$$

where $Z_{2}$ and $Z_{4}$ are the renormalization constants, $m_{0}$ is the current quark mass, $\Omega_{n l}=\omega_{n}-\omega_{l}$ is the gluon Matsubara frequency, $D_{\mu \nu}$ is the dressed-gluon propagator; and $\Gamma_{\nu}$ is the dressed-quark-gluon interaction vertex.

In our calculation, we take the rainbow approximation $\Gamma_{\nu}\left(\vec{q}, \tilde{\omega}_{l}, \vec{p}, \tilde{\omega}_{n}\right)=\gamma_{\nu}$ for the quark-gluon interaction vertex. The rainbow approximation is the leading order approximation [62,63]. It is essentially a mean field approximation [30-32], which will then lead to a clear analysis of the universality class.

To solve the gap equation, we should also have the information of the dressed-gluon propagator. The dressedgluon propagator is usually approximated as

$$
g^{2} D_{\mu \nu}\left(\vec{k}, \Omega_{n l}\right)=\mathcal{G}\left(k^{2}\right) D_{\mu \nu}^{\mathrm{free}}(k),
$$

where $D_{\mu \nu}^{\mathrm{free}}$ is the free gluon propagator 


$$
D_{\mu \nu}^{\mathrm{free}}(k)=\left(\delta_{\mu \nu}-\frac{k_{\mu} k_{\nu}}{k^{2}}\right) \frac{1}{k^{2}},
$$

and $k_{\mu}=\left(\vec{k}, \Omega_{n l}\right)$. The $\mathcal{G}\left(k^{2}\right)$ is the dressing function or, in other words, the effective interaction, which is usually represented in models. There have been now several models of the dressing function, for example, the Munczek-Nemirovsky model [56], the Roberts-Williams model [49,64], the Frank-Roberts model [65], the MarisTandy model [66], the Qin-Chang model [55], and contact models. In this paper, we will make use of the Qin-Chang model, the MN model, and two types of the contact model in our investigation.

At finite temperature, the gluon propagator should be split into longitudinal and transverse parts, which are in principle different from each other. In DSE model calculations, the difference of the two parts is usually taken into account by considering a gluon thermal mass in the longitudinal part; see, e.g., Refs. [40,67]. The inclusion of gluon thermal mass generally reduces the pseudocritical temperature, which has the same effect as the damping factor that will be introduced in Sec. II D. Therefore, in this work, we neglect the difference of the longitudinal and transverse parts of the gluon propagator and absorb its effect into the redefinition of the damping coefficient $\alpha$, defined in Eq. (20).

\section{A. Qin-Chang model}

In this paper, we make use of the QC model [55], which has not yet been applied in studies about the universality, even though it imitates in some sense the infrared constant behavior of QCD's gauge sector (see, e.g., Refs. [53,6875]) and reads

$$
\begin{aligned}
\frac{\mathcal{G}\left(k^{2}\right)}{k^{2}}= & 8 \pi^{2} D \frac{1}{\omega^{4}} e^{-k^{2} / \omega^{2}} \\
& +\frac{8 \pi^{2} \gamma_{m}}{\ln \left[\tau+\left(1+k^{2} / \Lambda_{\mathrm{QCD}}^{2}\right)^{2}\right]} \mathcal{F}\left(k^{2}\right),
\end{aligned}
$$

where $\mathcal{F}\left(k^{2}\right)=\left(1-\exp \left(-k^{2} / 4 m_{t}^{2}\right) / k^{2}, \tau=e^{2}-1, m_{t}=\right.$ $0.5 \mathrm{GeV}, \Lambda_{\mathrm{QCD}}=0.234 \mathrm{GeV}$, and $\gamma_{m}=12 /\left(33-2 N_{f}\right)$ with $N_{f}$ the number of flavors. We need to notice that, although we will only solve the quark DSE with small current quark mass corresponding to $u$ and $d$ quarks, the solution is actually the $u / d$ sector for a system consisting of more quark flavors. Therefore, we take $N_{f}=4$ in our practical calculations, since the top and bottom quarks are too heavy to be considered.

This gluon propagator leads to an ultraviolet divergence for the integration in Eq. (3). To deal with that, we take the renormalization scheme as that used in Refs. [76,77]. In vacuum, the summation over the Matsubara frequency in Eq. (3) converts in to an integration over 4-momentum, and we have $A\left(p^{2}\right)=C\left(p^{2}\right)$ because of the Lorentz symmetry. The renormalization condition in vacuum is

$$
\begin{aligned}
\left.A\left(p^{2}\right)\right|_{m_{0}=0, p^{2}=\zeta^{2}, T=0, \mu=0} & =1, \\
\left.\frac{\partial B\left(p^{2}\right)}{\partial m_{0}}\right|_{m_{0}=0, p^{2}=\zeta^{2}, T=0, \mu=0} & =1,
\end{aligned}
$$

where $A\left(p^{2}\right)$ and $B\left(p^{2}\right)$ are evaluated in vacuum and in chiral limit, $m_{0}$ is the current quark mass, and $\zeta$ is the renormalization point. Using Eq. (7), we can calculate the renormalization constant $Z_{2}$ and $Z_{4}$ in vacuum and use them to solve the DSE at finite temperature. Notice that the renormalization constants are independent of the current quark mass under this scheme.

There are two parameters, $D$ and $\omega$ in the QC model. In this work, we will take $(D \omega)^{1 / 3}=0.82 \mathrm{GeV}$ and $\omega=$ $0.5 \mathrm{GeV}$ together with light quark mass $m_{0}=6.6 \mathrm{MeV}$, for renormalization point $\zeta=2 \mathrm{GeV}$, which reproduces pion properties $m_{\pi}=0.14 \mathrm{GeV}$ and $f_{\pi}=0.092 \mathrm{GeV}$ [77].

In numerical realization, we also need a cutoff for the upper boundary of the 3-momentum integration and Matsubara frequency summation in Eq. (3). In this work, we take $\Lambda_{|\vec{p}|}=1000 \mathrm{GeV}$ and $n_{\max }=50$ in order to guarantee the precision and efficiency simultaneously. We also notice that the result will not change much as long as $\Lambda_{|\vec{p}|}$ and $\omega_{n_{\max }}$ is greater than the renormalization point $\zeta$.

\section{B. Munczek-Nemirovsky model}

Noticing that the function $\mathcal{G}\left(k^{2}\right)$ in Eq. (6) is served as a momentum distribution of interaction, this model could cover various types of the interaction via varying the $\omega$, which stands for the interaction length of QCD. Except for the perturbative correction, as $\omega$ goes to 0 , the distribution becomes a $\delta$-function, which is just the so-called MunczekNemirovsky model $[56,78]$.

At finite temperature, the MN model could be generalized as

$g^{2} D_{\mu \nu}\left(\vec{p}, \Omega_{k}\right)=\left(\delta_{\mu \nu}-\frac{p_{\mu} p_{\nu}}{|\vec{p}|^{2}+\Omega_{k}^{2}}\right) 2 \pi^{3} \frac{\eta^{2}}{T} \delta_{k 0} \delta^{3}(\vec{p})$,

where $\Omega_{k}=2 k \pi T$ is the boson Matsubara frequency and $\left(p_{\mu}\right)=\left(\vec{p}, \Omega_{k}\right) \cdot \eta$ is a mass-scale parameter. Using this gluon model, the integration in DSE equations can be completed analytically, and the DSE becomes [78]

$$
\begin{aligned}
\eta^{2} m_{0}^{2}= & B^{4}+m_{0} B^{2}+\left(4 \tilde{p}_{k}^{2}-\eta^{2}-m_{0}^{2}\right) B^{2} \\
& -m_{0}\left(2 \eta^{2}+m_{0}^{2}+4 \tilde{p}_{k}^{2}\right) B \\
A\left(\tilde{p}_{k}\right)= & C\left(\tilde{p}_{k}\right)=\frac{2 B\left(\tilde{p}_{k}\right)}{m_{0}+B\left(\tilde{p}_{k}\right)} .
\end{aligned}
$$

Reference [78] shows that with parameters $\eta=$ $1.37 \mathrm{GeV}$ and $m_{0}=30 \mathrm{MeV}$, one can get the pion mass $m_{\pi}=140 \mathrm{MeV}$. 


\section{Contact models}

\section{Contact model in Landau gauge}

In Eq. (6), if we increase the $\omega$ while keeping $8 \pi^{2} \frac{D}{\omega^{4}}=$ $\frac{1}{m_{G}^{2}}$ as a constant, the gluon propagator becomes (neglecting the perturbation term)

$$
g^{2} D_{\mu \nu}=\frac{1}{m_{G}^{2}}\left(\delta_{\mu \nu}-\frac{k_{\mu} k_{\nu}}{k^{2}}\right) .
$$

This model is the contact model in Landau gauge. It is the antithetical complement of the MN model and is similar to the Nambu-Jona-Lasinio (NJL) model since the interaction mainly appears when the separation $x=0$.

If we adopt this model, the integration in Eq. (3) will be drastically divergent, and the theory is not renormalizable. In this work, we will use a sharp 3-momentum cutoff to do the regularization, which is often done for the NJL model (see, for example, the review of Ref. [79]). Notice that this regularization scheme will break the $\mathcal{O}(4)$ Lorentz symmetry explicitly in vacuum. However, it is not a severe problem, especially at finite temperature since the symmetry is broken by the medium anyway.

In NJL calculations, the 3-momentum cutoff is a model parameter, and it ranges around $500-700 \mathrm{MeV}$; see, e.g., Table 2.2 and Table 3.1 in Ref. [79]. Therefore, for the contact model in Landau gauge, we choose the 3-momentum cutoff $|\vec{\Lambda}|=0.6 \mathrm{GeV}$ in analogy of that in the NJL model. The cutoff on the Matsubara frequency is chosen to be $n_{\max }=50$, which is the same as in the QC model. Then, we extend the approximation formula $[57,58]$ to fix the gluon mass $m_{G}$ and current quark mass $m_{0}$ (for the derivations, please refer to the Appendix A), which reads

$m_{\pi}^{2} N_{\pi}^{2}=8 N_{c} \int \frac{\mathrm{d}^{4} p}{(2 \pi)^{4}} B_{m_{0}=0}\left[\sigma_{B}^{m_{0}=0}-\frac{B_{m_{0}=0}}{B_{m_{0} \neq 0}} \sigma_{m_{0} \neq 0}^{B}\right]$,

where $B_{m_{0}=0}=B_{m_{0}=0}\left(\vec{p}^{2}, p_{4}^{2}\right)$ is the scalar function for the quark propagator in vacuum at chiral limit, and

$\sigma^{F}\left(\vec{p}^{2}, p_{4}^{2}\right)=\frac{F\left(\vec{p}^{2}, p_{4}^{2}\right)}{\vec{p}^{2} A^{2}\left(\vec{p}^{2}, p_{4}^{2}\right)+p_{4}^{2} C^{2}\left(\vec{p}^{2}, p_{4}^{2}\right)+B^{2}\left(\vec{p}^{2}, p_{4}^{2}\right)}$,

where $F=A, B, C$.

In Eq. (11), $N_{\pi}$ is the normalization factor, and its approximation is

$$
\begin{aligned}
N_{\pi}^{2}= & 8 N_{c} \int \frac{\mathrm{d}^{4} p}{(2 \pi)^{4}} B_{m_{0}=0}^{2} \\
& \times\left\{-\frac{1}{4}\left(\sigma^{C}\right)^{2}+\vec{p}^{2}\left[\frac{1}{2} \sigma^{A} \sigma_{4}^{A}+p_{4}^{2} \sigma^{A} \sigma_{44}^{A}-2 p_{4}^{2}\left(\sigma_{4}^{A}\right)^{2}\right]\right. \\
& +\frac{1}{2} \sigma^{B} \sigma_{4}^{B}+p_{4}^{2} \sigma^{B} \sigma_{44}^{B}-2 p_{4}^{2}\left(\sigma_{4}^{B}\right)^{2} \\
& \left.+p_{4}^{2}\left[\frac{1}{2} \sigma^{C} \sigma_{4}^{C}+p_{4}^{2} \sigma^{C} \sigma_{44}^{C}-2 p_{4}^{2}\left(\sigma_{4}^{C}\right)^{2}\right]\right\}
\end{aligned}
$$

where $\sigma_{4}^{F}=\frac{\partial \sigma^{F}}{\partial p_{4}^{2}}$ and $\sigma_{44}^{F}=\frac{\partial^{2} \sigma^{F}}{\partial\left(p_{4}^{2}\right)^{2}}$ for $F=A, B, C$.

The pion decay constant $f_{\pi}$ can be approximately determined with the Gell-Mann-Oakes-Renner (GOR) relation,

$$
f_{\pi}^{2} m_{\pi}^{2}=2 m_{0}\langle\bar{q} q\rangle_{m_{0}=0}
$$

we have then

$m_{\pi}^{2} f_{\pi}^{2}=8 N_{c} \int \frac{\mathrm{d}^{4} p}{(2 \pi)^{4}} \frac{m_{0} B_{m_{0}=0}}{p_{4}^{2} C_{m_{0}=0}^{2}+\vec{p}^{2} A_{m_{0}=0}^{2}+B_{m_{0}=0}^{2}}$.

The fitted parameter is $m_{G}=0.1 \mathrm{GeV}$ and $m_{0}=$ $3.7 \mathrm{MeV}$ for $m_{\pi}=140 \mathrm{MeV}$ and $f_{\pi}=92 \mathrm{MeV}$. For simplicity, in the following, we will use "contact L" to denote the contact model in Landau gauge.

\section{Roberts's contact model}

The contact model in Landau gauge can be directly derived from the QC model. However, if we use another gauge, we can accomplish the integration in Eq. (3) and get an analytic result. Such a contact model which is more widely used has the form $[59,60,80,81]$

$$
g^{2} D_{\mu \nu}=\delta_{\mu \nu} \frac{1}{m_{G}^{2}} .
$$

With this simple gluon model, we have $A(p)=C(p)=1$, and $B(p)=M$ is momentum independent. The DSE can be simplified to

$$
M=m_{0}+\frac{16}{3} T \frac{1}{m_{G}^{2}} \sum_{n} \int \frac{\mathrm{d}^{3} \vec{q}}{(2 \pi)^{3}} \frac{M}{\vec{q}^{2}+[(2 n+1) \pi T]^{2}+M^{2}} .
$$

For the regularization, following the scheme in Ref. [60], we have

$$
\frac{1}{s+M^{2}}=\int \mathrm{d} \tau e^{-\tau\left(s+M^{2}\right)} \rightarrow \int_{\tau_{\mathrm{UV}}^{2}}^{\tau_{\mathrm{IR}} 2} \mathrm{~d} \tau e^{-\tau\left(s+M^{2}\right)} .
$$


Then, the summation over the Matsubara frequency can be done, and the DSE becomes

$$
M=m_{0}+\frac{M}{3 \pi^{2}} \frac{1}{m_{\mathrm{G}}^{2}} \int_{\tau_{\mathrm{UV}}^{2}}^{\tau_{\mathrm{IR}}^{2}} d \tau \frac{e^{-\tau M^{2}} \vartheta_{3}\left(\frac{\pi}{2}, e^{-\frac{1}{4 \tau T^{2}}}\right)}{\tau^{2}}
$$

where $\vartheta_{a}(u, x)$ is the Jacobian theta function.

In this paper, we are going to take $m_{G}=0.132 \mathrm{GeV}$, $\tau_{\mathrm{UV}}=1 / 0.905 \mathrm{GeV}^{-1}$, and $\tau_{\mathrm{IR}}=1 / 0.24 \mathrm{GeV}^{-1}$, with which the pion mass and decay constant $m_{\pi}=$ $0.140 \mathrm{GeV}$ and $f_{\pi}=0.101 \mathrm{GeV}$ are obtained [60]. For simplicity, in the following, we will use "contact R" to denote Roberts's contact model.

\section{Damping factor}

In practical calculations, the parameters for the gluon models above are fixed by meson properties in vacuum. However, as we know, QCD is an asymptotic free theory, and the interaction should be weak and approaches zero at high temperature. The parameters should then be modified at finite temperature.

For the QC model, we do the modification simply by multiplying a damping factor to the coupling constant $D$,

$$
D(T)=D \times \frac{1}{\left(1+\alpha \frac{T}{m_{\pi}}\right)^{2}},
$$

where $\alpha$ is the parameter which controls the speed for the interaction to approach zero. $m_{\pi}=140 \mathrm{MeV}$ is the physical pion mass, and we take it here only to make $\alpha$ dimensionless. For the MN model and the contact models, the same expression of the damping factor $1 /(1+$ $\left.\alpha T / m_{\pi}\right)^{2}$ can be directly taken to the dressed-gluon propagator.

The dimensionless damping parameter $\alpha$ is calibrated to reproduce the pseudocritical temperature $T_{c}=156.5 \mathrm{MeV}$ at physical pion mass [29]. The corresponding values of $\alpha$

TABLE I. Parameters and fitted results for different dressing functions. $m_{\text {phy }}$ is the current quark mass corresponding to physical pion mass. $\alpha$ is the damping parameter. $T_{c, 0}$ is the critical temperature for the phase transition in chiral limit. $C$ is the coefficient for fitting the pion mass, defined in Eq. (23). The last line is the percentage of maximum chiral susceptibility which corresponds to a quark-mass-independent pseudocritical temperature. See the text for detail.

\begin{tabular}{lcccc}
\hline \hline Model & QC & MN & Contact L & Contact R \\
\hline$m_{\text {phy }}(\mathrm{MeV})$ & 6.6 & 30 & 3.7 & 7.0 \\
$\alpha$ & 0.1731 & 0.5274 & 0.4900 & 0.1744 \\
$T_{c, 0}(\mathrm{MeV})$ & 143.6 & 142.0 & 152.2 & 142.9 \\
$C\left(\mathrm{MeV}^{1 / 2}\right)$ & 54.49 & 25.56 & 73.40 & 52.76 \\
Percentage & 78 & 80 & 80 & 79 \\
\hline \hline
\end{tabular}

for different dressing functions are listed in the second line of Table I.

The choice of the damping factor formula is arbitrary as long as the formula produce the respectively same value at $T=0$ and $T=156.5 \mathrm{MeV}$ for each of the gluon dressing functions. However, in this paper, we are interested in the relation between the pseudocritical temperature and the current quark mass. And the temperature range we study is only $\Delta_{T}=T_{c}\left(m_{0}=m_{\text {phy }}\right)-T_{c}\left(m_{0}=0\right) \sim 10 \mathrm{MeV}$. Therefore, the effect of the damping factor is mainly determined by its value at $T=T_{c}$, which is irrelevant to the choice of the damping factor formula, as long as the function is not too stiff in terms of the temperature $T$.

\section{E. Chiral susceptibility}

The chiral symmetry property manifests by the quark propagator straightforwardly. In chiral limit, the order parameter of chiral symmetry is usually defined as the quark condensate, which is an integral of the trace of quark propagator (see, e.g. Refs. [50,82]). At finite current quark mass, however, since the integral is divergent, a direct integration is ill-defined. Therefore, there are several different ways to eliminate the divergence of the quark condensate $[12,29,40,83-86]$. Besides, noticing that the dominant contribution in the ultraviolet region is perturbative due to the asymptotic free behavior, and the DCSB effect is generated mainly by the infrared domain of the interaction, the order parameter could also be interpreted as the running mass of the quark at zero momentum. Different definitions of the order parameter will not change the phase diagram for the first and second order phase transitions and may change slightly the location of the pseudocritical temperature in crossover region (see, e.g., Ref. [40]). However, the critical exponents are universal and will not be altered. Therefore, in this paper, we take simply the $B\left(\omega_{0}, \vec{p}=0\right)$ as the order parameter.

After that, the chiral susceptibility could be defined as the derivative with respective to the current quark mass $[7,11,29]$ :

$$
\chi=\frac{\partial B\left(\omega_{0}, \vec{p}=0\right)}{\partial m_{0}}
$$

The pseudocritical temperature is then just the temperature for the $\chi$ to reach its maximum. Such a definition of the chiral susceptibility is not exactly the same as that used in lattice QCD and functional renormalization group calculations; however, the difference between the extracted pseudocritical temperature should be quite limited [40]. Therefore, the exact value of chiral susceptibility in this paper is different from those in Refs. [11,29], but the obtained pseudocritical temperatures are comparable (see the next section). 


\section{RESULTS AND DISCUSSIONS}

In this section, we present our numerical results calculated by the DSE with the various gluon models mentioned above. In Fig. 1, we show the chiral susceptibility as a function of temperature for several values of the current quark mass $m_{0}$ in the QC model. It is evident that the peak gets narrower and narrower as the current quark mass becomes very small. At the chiral limit, the chiral susceptibility becomes divergent, which means it becomes the second order phase transition [7]. The calculated chiral susceptibilities for the other models have the similar behavior; we then omit the corresponding figures to save the length of the paper.

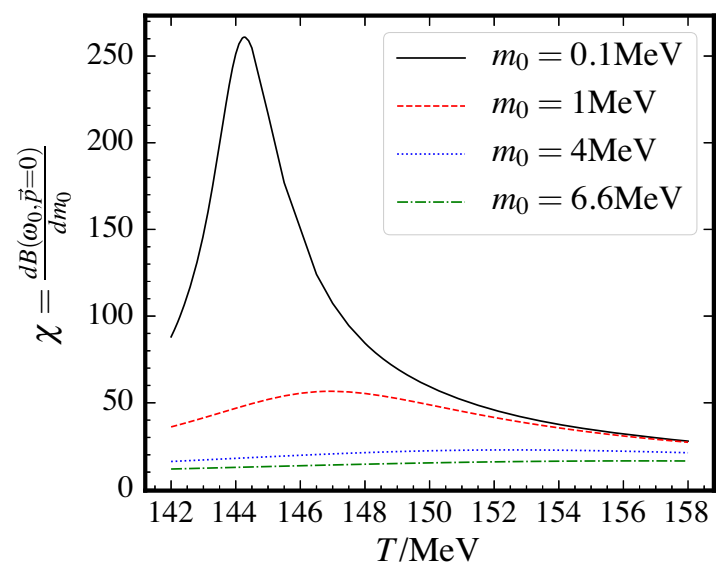

FIG. 1. Calculated chiral susceptibility as a function of temperature for several current quark masses.

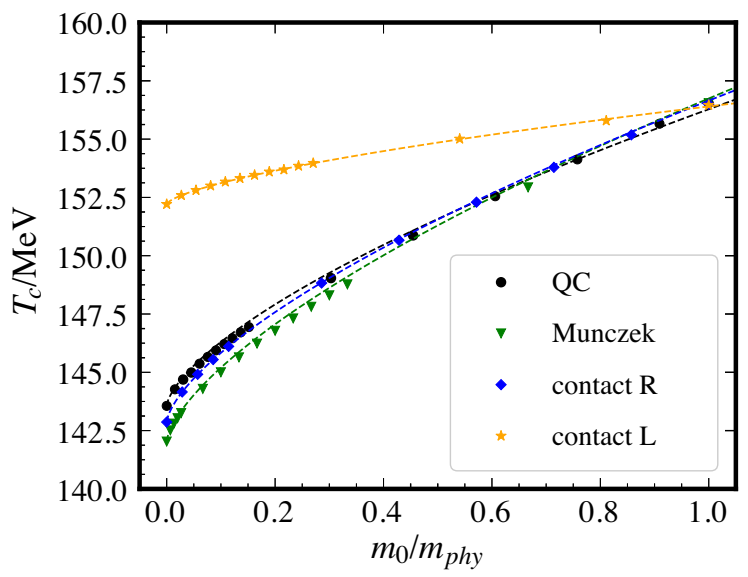

FIG. 2. Calculated pseudocritical temperature as a function of current quark mass rescaled by $m_{\text {phy }}$, which is the quark mass corresponding to the physical pion mass $m_{\pi}=140 \mathrm{MeV}$. The different symbols with different colors stand for the results calculated via different models. The dashed lines correspond to the fit of the calculated data using the formula $T=a m_{0}^{2 / 3}+T_{c}^{\text {chiral }}$. Contact $\mathrm{R}$ in the legend means Roberts's contact model used in Refs. [59,60], and contact L represents the contact model in Landau gauge described in Sec. II.
In Fig. 2, we present the calculated results of the pseudocritical temperature as a function of the current quark mass via the four models of the gluon dressing function. The current quark mass corresponding to the physical pion mass is $m_{\text {phy }}=6.6 \mathrm{MeV}$ for the QC model [77], $m_{\text {phy }}=30 \mathrm{MeV}$ for the $\mathrm{MN}$ model [78], $m_{\text {phy }}=$ 3.7 $\mathrm{MeV}$ for the contact model in Landau gauge, and $m_{\text {phy }}=7 \mathrm{MeV}$ for Roberts's contact model [60]. These values are listed in the first line of Table I. The pseudocritical temperature for all the models have been tuned to 156.5 MeV by altering the parameter $\alpha$ in Eq. (20). The obtained pseudocritical temperature at the chiral limit is shown in the third line of Table I, which is $T_{c}=$ 143.6 MeV for the QC model, $142.0 \mathrm{MeV}$ for the MN model, $152.2 \mathrm{MeV}$ for the contact model in Landau gauge, and 142.9 MeV for Roberts's contact model.

In order to study further the behavior of the phase transition, we take a formula $T=a m_{0}^{b}+c$ to fit the calculated data. We fix $c=T_{c}^{\text {chiral }}$ with $T_{c}^{\text {chiral }}$ the pseudocritical temperature since there is no ambiguity for our result of the pseudocritical temperature in the chiral limit (i.e., in case of $\left.m_{0}=0\right)$ as the susceptibility becomes divergent. For the power index $b$, we could make use of the value of the critical exponent as $b=1 /(\beta \delta)=2 / 3$ in our mean field approximation, which is consistent with the $3 \mathrm{D} \mathrm{O}(4)$ universality class $[31,39]$. As can be seen from the figure, the calculated result can be fitted quite well with the mean field critical exponent. Such a result is also consistent with that given by analyzing the heat capacity in the DSE approach of QCD [28].

Moreover, we compute the scaling behavior of the chiral susceptibility with respect to the current quark mass. The universality class analysis gives that [31]

$$
\chi=A m_{0}^{-(1-1 / \delta)} .
$$

In Fig. 3, we illustrate the calculated data and the fitted result. It is apparent that we can assign the parameter as $\delta=3$, which is consistent with 3D O(4) universality class. In some details, we can extract that the scaling behavior is only valid up to $m_{0} \lesssim 0.5 \mathrm{MeV}$ for the contact model in Landau gauge, while $m_{0} \lesssim 1-2 \mathrm{MeV}$ for the other three models. Notice that, since the physical current quark mass is larger in the $\mathrm{MN}$ model, the critical region for the $\mathrm{MN}$ model is actually smaller than the other models. After analyzing the scaling behavior of the chiral susceptibility with respect to temperature, we get another critical exponent as $\beta=1 / 2$.

The scaling behavior could also be demonstrated in terms of the mass of pion since the pion mass is an experimental quantity, and it is better than that of the current quark mass, which is dependent on the renormalization schemes. It has been well known that the GOR relation demonstrates a simple connection between the pion mass and the current quark mass near the chiral limit, which 


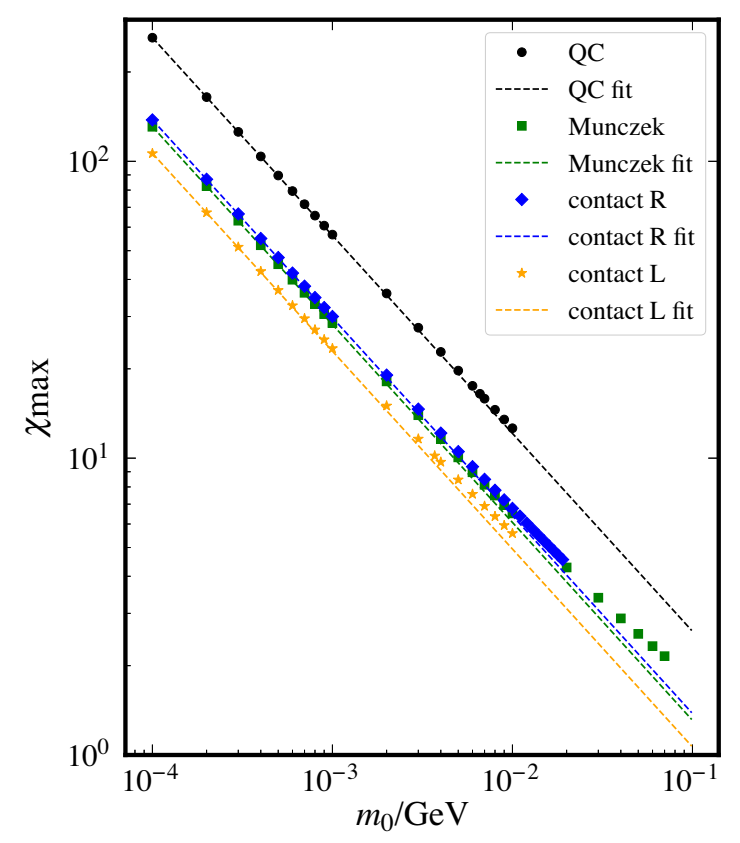

FIG. 3. Calculated chiral susceptibility as a function of current quark mass. The dashed lines represent the fitted results of the calculated data using the formula $\chi=A m_{0}^{-(1-1 / \delta)}$ with $\delta=3$.

has been shown in Eq. (14). In this paper, we can then take the leading order of this relation and have

$$
m_{\pi}=C \sqrt{m_{0}}, \quad \text { in the unit of } \mathrm{MeV},
$$

where $C$ is a coefficient depending on models, with the unit $\mathrm{MeV}^{1 / 2}$. The value of $C$ can be determined by fitting the results given by the Bethe-Salpeter (BS) equation calculation, and the obtained results read $54.49 \mathrm{MeV}^{1 / 2}$ for the QC model [77], $25.56 \mathrm{MeV}^{1 / 2}$ for the MN model [78], $52.76 \mathrm{MeV}^{1 / 2}$ for Roberts's contact model [60], and $73.04 \mathrm{MeV}^{1 / 2}$ for the contact model in Landau gauge.

The calculated result of the $T_{c}$ as a function of $m_{\pi}$ is shown in Fig. 4. For comparison, we plot also the result obtained with the functional renormalization group approach of QCD (fQCD) [29] and that given in lattice QCD (HotQCD) [11,29]. As can be seen from the figure, in contrast to the linear dependence from the fQCD and HotQCD results, in our calculation, the $T_{c}$ has an approximately $4 / 3$ power dependence on the pion mass. Beyond the critical region, we can infer from the figure that the relation between $T_{c}$ and $m_{\pi}$ goes gradually to a simple linear relation. Recalling that the critical exponent of the 3D O(4) class beyond the mean field is around 1 [11,29], one can infer that the full QCD computation could find a linear relation between $T_{c}$ and $m_{\pi}$ without the limitation of critical region.

In lattice QCD simulation, it has been argued that the temperature at which the chiral susceptibility is $60 \%$ of its maximum remains nearly the same for a large range of

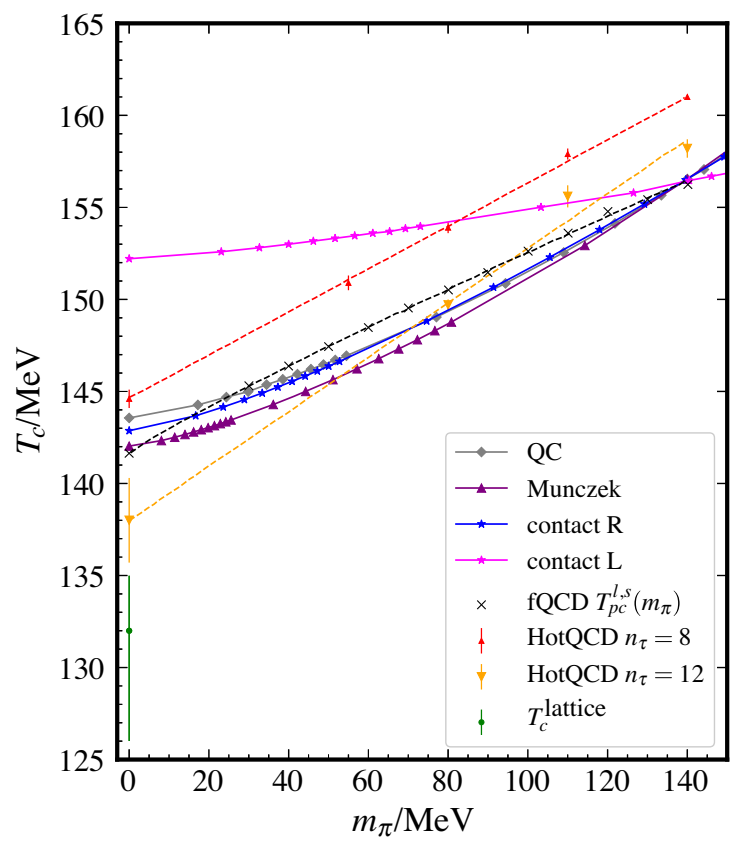

FIG. 4. Calculated pseudocritical temperature as a function of pion mass. The fQCD result is taken from Ref. [29], and the HotQCD result is taken from Ref. [11]. $T_{c}^{\text {lattice }}$ is the lattice QCD result of the chiral phase transition after thermodynamic, continuum, and chiral extrapolations [11].

quark mass, so that the critical temperature for the chiral symmetry to be restored in chiral limit is obtained by extrapolation [11]. In order to investigate such an extrapolation scheme, we present the calculated pseudocritical

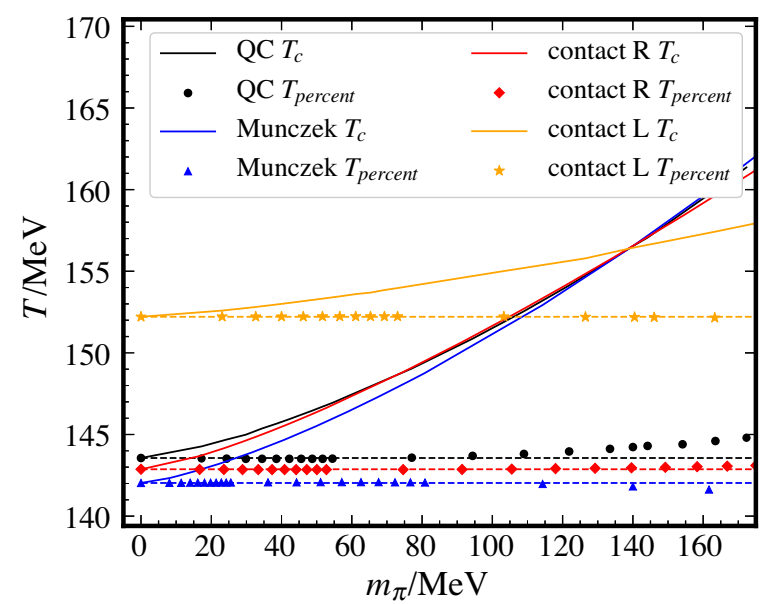

FIG. 5. Comparison of the calculated pion mass dependence of the typical pseudocritical temperature $\left(T_{c}\right)$, the extrapolated pseudocritical temperature ( $\left.T_{\text {percent }}\right)$, and the calculated pseudocritical temperature in chiral limit, in the four models. The solid line corresponds to $T_{c}$. The dotted lines with different symbols stand for the $T_{\text {percent }}$ with the percentage being $78 \%, 80 \%, 79 \%$, and $80 \%$ for the QC model, the MN model, Roberts's contact model, and the contact model in Landau gauge, respectively. The horizontal dashed lines represent the $T_{\text {chiral }}$ in the four models. 
temperature with a certain percentage of the maximal susceptibility via the different gluon models in Fig. 5. The solid lines in Fig. 5 correspond to a percentage of $100 \%$; i.e., they correspond to the typical pseudocritical temperature $T_{c}$. The horizontal dashed lines stand for the pseudocritical temperature in the chiral limit, $T_{\text {chiral }}$. The symbols $\bullet, \mathbf{\Delta}, \downarrow$, and $\star$ represents the temperature corresponding to a percentage of the maximal chiral susceptibility, $T_{\text {percent }}$, in the QC, MN, contact $R$, and contact $L$ model, respectively, which will be denoted as the extrapolated pseudo-critical temperature.

In our calculation, however, if we take $60 \% \chi_{\max }$ to be a criterion, the pseudocritical temperature will not be constant for different current quark mass. However, when we survey the obtained results with various percentages carefully, we observe that there exists a pion mass range where $T_{\text {percent }}$ remains almost invariable. The obtained percentage for the pion mass range to reach its maximum is $78 \%, 80 \%$, $79 \%$, and $80 \%$ for the QC model, the MN model, Roberts's contact model, and the contact model in Landau gauge, respectively. As we can see from Fig. 5, for the contact model in Landau gauge, although there exists an systematic deviation, we have $\left|T_{\text {percent }}-T_{\text {chiral }}\right|<1 \mathrm{MeV}$ even at the largest pion mass we calculated, and this difference can hardly be seen on the figure. For the QC, the MN, and Roberts's contact model, however, when the pion mass (and the current quark mass) is large enough, the $T_{\text {percent }}$ deviates from the horizontal dashed line, $T_{\text {chiral }}$, in the corresponding model. The $T_{\text {percent }}$ begins to increase at $m_{\pi} \sim 100 \mathrm{MeV}$ in the QC model, while the $T_{\text {percent }}$ remains unchanged up to physical pion mass for the contact model and the MN model.

\section{CONCLUSIONS}

It has been argued that the phase transition of QCD at the chiral limit belongs to the 3D O(4) universality class. The universality class analysis gives the critical behavior at the chiral limit as that relation between the pseudocritical temperature and the current quark mass behaves as $T_{c} \sim m_{0}^{1 / \beta \delta}$. In this paper, we calculate and analyze the dependence of the pseudocritical temperature on the current quark mass in the framework of the DS equation approach of QCD with the rainbow truncation and several different models for the dressed gluon. We find that our result is very consistent with the universality class result, and the critical exponent for the different gluon models is the same as that of the mean field result.

We have implemented four different types of dressedgluon models, which represent a large range of momentum distribution of the interaction kernel, three of which were fully investigated before, and we also considered the contact model in Landau gauge for comparison. Our calculated results show that the mean field behavior is not affected by the interaction kernel. There are several possible ways to get results beyond mean field approximation. For example, one can take the quark-gluon vertex model with complicated tensor structures [15,87-92], or consider the pion-exchange effect on the Bethe-Salpeter kernel which also contains the mass dependence via pion mass [33], or take into account the baryon effect [93], even the more theoretical ones(see, e.g., Refs. [94,95]). These calculations will be done in our further study.

We analyze the scaling behavior with different features of interaction by considering different dressed-gluon models. The obtained critical exponent is consistent with that of the 3D O(4) universality class. The critical region is up to $m_{0} \sim$ $1-2 \mathrm{MeV}$ in this mean field approximation, which sets naturally an upper bound of the critical region since the fluctuations beyond the mean field would usually diminish the critical region even break the critical behavior.

Another interesting quantity that has been marked by lattice QCD simulation analysis is the pseudocritical temperature where the chiral susceptibility is the $60 \%$ of the maximum, which is found to be independent of the current quark mass. However, our results show that this is only true when the current quark mass and the pion mass are not too large. For the QC model, this temperature is invariant only for $m_{\pi} \lesssim 100 \mathrm{MeV}$, while for the contact and the MN model, it can be invariant up to the physical pion mass. The fixed percentage of the maximal chiral susceptibility is different for different models.

\section{ACKNOWLEDGMENTS}

The work was supported by the National Natural Science Foundation of China under Contracts No. 11435001, No. 11775041, No. 11947108, and No. 11605254. F. G. is grateful for the support from Alexander von Humbodlt Foundation.

\section{APPENDIX: APPROXIMATION FORMULA FOR PION MASS AND DECAY CONSTANT}

The pion mass and the decay constant should be calculated by solving the Bethe-Salpeter (BS) equation. However, for the bare vertex, an approximation formula with good accuracy has been introduced in Ref. [57]. In this paper, we are going to take this approximation to determine the gluon mass and physical current quark mass of the contact $\mathrm{L}$ model, introduced in Sec. II C 1. Since this model needs to break the $O(4)$ symmetry by hand in vacuum, we need to make some modifications to the approximation formula.

Under the rainbow-ladder truncation, the homogeneous BS equation reads

$$
\begin{aligned}
\Gamma(k ; P)= & -\frac{4}{3} Z_{2}^{2} \int \frac{\mathrm{d}^{4} q}{(2 \pi)^{4}}\left[\mathcal{G}\left((k-q)^{2}\right) D_{\alpha \beta}^{\mathrm{free}}(k-q)\right. \\
& \left.\times \gamma_{\alpha} S\left(q_{+}\right) \Gamma(q ; P) S\left(q_{-}\right) \gamma_{\beta}\right],
\end{aligned}
$$

where $\Gamma$ is the Bethe-Salpeter amplitude, $q_{ \pm}=q \pm \frac{1}{2} P$, and $P$ is the momentum of the meson. $Z_{2}$ is the 
renormalization constant, and we will omit this factor since its value can be absorbed into the parameter of the effective interaction. We notice that, by omitting the renormalization constant, the model will be cutoff dependent, and this cutoff can also be regarded as a parameter of the model. A model parameter $|\vec{\Lambda}|$ appears then in the contact L model as mentioned in the main text.

The BS amplitude $\Gamma$ has complicated Lorentz structure for different mesons. But for a pion, it is a good approximation to write

$$
\Gamma=i \gamma_{5} B_{m_{0}=0}\left(|\vec{p}|, p_{4}\right),
$$

where $B_{m_{0}}=0$ is the Lorentz scalar part of the quark propagator at chiral limit. The BS equation then becomes

$$
\begin{aligned}
i \gamma_{5} B_{m_{0}=0}\left(|\vec{p}|, p_{4}\right)= & -i \frac{4}{3} \int \frac{\mathrm{d}^{4} q}{(2 \pi)^{4}} B_{m_{0}=0}\left(|\vec{q}|, q_{4}\right) \frac{\mathcal{G}\left(l^{2}\right)}{l^{2}} \\
& \times\left(\delta_{\mu \nu}-\frac{l_{\mu} l_{\nu}}{l^{2}}\right) \gamma_{\alpha} S\left(q_{+}\right) \gamma_{5} S\left(q_{-}\right) \gamma_{\beta},
\end{aligned}
$$

where $l=p-q$.

By multiplying $\gamma_{5}$ and taking trace on both sides of the equation, we have

$$
\begin{aligned}
& B_{m_{0}=0}\left(|\vec{p}|, p_{4}\right) \\
& =4 \int \frac{\mathrm{d}^{4} q}{(2 \pi)^{4}} B_{m_{0}=0}\left(|\vec{p}|, p_{4}\right) \frac{G\left(l^{2}\right)}{l^{2}} \\
& \quad \times\left[\sigma_{A}^{+} \sigma_{A}^{-}\left(\vec{q}_{+} \cdot \vec{q}_{-}\right)+\sigma_{B}^{+} \sigma_{B}^{-}+\sigma_{C}^{+} \sigma_{C}^{-}\left(q_{4+} q_{4-}\right)\right],
\end{aligned}
$$

where $\vec{q}_{ \pm}=\vec{q} \pm \frac{1}{2} \vec{P}, q_{4 \pm}=q_{4} \pm \frac{1}{2} P_{4}$, and

$\sigma_{F}^{ \pm}=\frac{F\left(\vec{p}_{ \pm}^{2}, p_{4 \pm}^{2}\right)}{\vec{p}_{ \pm}^{2} A^{2}\left(\vec{p}_{ \pm}^{2}, p_{4 \pm}^{2}\right)+B^{2}\left(\vec{p}_{ \pm}^{2}, p_{4 \pm}\right)+p_{4 \pm}^{2} C^{2}\left(\vec{p}_{ \pm}^{2}, p_{4 \pm}^{2}\right)}$

for $F=A, B, C$.

Defining $\Delta(l)=\mathcal{G}\left(l^{2}\right) / l^{2}$, one can rewrite the Eq. (A4) as

$$
8 N_{c} B_{m_{0}=0}\left(p^{2} ; P^{2}\right)=3 C_{2}(R) \int \frac{\mathrm{d}^{4} q}{(2 \pi)^{4}} \Delta(p-q) H(q ; P),
$$

where $C_{2}(R)=4 / 3$ and

$$
\begin{aligned}
H(p ; P)= & 8 N_{c}\left[\sigma_{A}^{+} \sigma_{A}^{-}\left(\vec{q}_{+} \cdot \vec{q}_{-}\right)\right. \\
& \left.+\sigma_{B}^{+} \sigma_{B}^{-}+\sigma_{C}^{+} \sigma_{C}^{-}\left(q_{4+} q_{4-}\right)\right] B_{m_{0}=0}\left(|\vec{p}|, p_{4}\right) .
\end{aligned}
$$

Equation (A6) is a convolution, and it can be simplified by Fourier transformation,

$$
8 N_{c} B_{m_{0}=0}(x)=3 C_{2}(R) \Delta(x) H_{P}(x) .
$$

By multiplying $F_{P}(-x) /\left[3 C_{2}(R) \Delta(x)\right]$, we can construct

$\Pi_{\pi}(P) \equiv \int \mathrm{d}^{4} x\left(\frac{8 N_{c}}{3 C_{2}(R)} \frac{F_{P}(-x) F_{P}(x)}{\Delta(x)}-F_{P}(-x) H_{P}(x)\right)$,

where $\Pi_{\pi}$ serves as the inverse of the pion propagator $[57,96]$.

Therefore, the pole of $\Pi_{\pi}$ indicates the mass of the meson, and our task now is to find the $m_{\pi}$ which satisfies

$$
\Pi_{\pi}\left(P^{2}=-m_{\pi}^{2}\right)=0 .
$$

We notice that the DSE for $B\left(\vec{p}^{2}, p_{4}^{2}\right)$ is also a convolution, and we have

$$
B_{m_{0}=0}(x)=3 C_{2}(R) \Delta(x) \sigma_{B}^{m_{0}=0}(x) .
$$

And we can obtain

$\Pi_{\pi}(P)=\int \mathrm{d}^{4} x B_{m_{0}=0}(x)\left[8 N_{c} \sigma_{S}^{m_{f}=0}-H_{P}\left(x ; m_{0}\right)\right]$.

Since the pion has a small mass, we can write

$$
\Pi_{\pi}(P)=\Pi_{\pi}(0)+|\vec{P}|^{2}\left(\frac{\partial \Pi_{\pi}}{\partial \vec{P}^{2}}\right)_{P^{2}=0}+P_{4}^{2}\left(\frac{\partial \Pi_{\pi}}{\partial P_{4}^{2}}\right)_{P^{2}=0} .
$$

From now on, we assume that the pion is in the rest frame, and the 4-momentum is $P=(\overrightarrow{0}, i M)$. We have then

$$
\Pi_{\pi}(P)=\Pi_{\pi}(0)+P_{4}^{2}\left(\frac{\partial \Pi_{\pi}}{\partial P_{4}^{2}}\right)_{P^{2}=0} .
$$

The $N_{\pi}^{2}$ can be defined as

$$
N_{\pi}^{2}=\left(\frac{\partial \Pi_{\pi}}{\partial P_{4}^{2}}\right)_{P^{2}=0}
$$

Differentiating the $\Pi_{\pi}$, we can get the formula we use in Eq. (13). 
By definition, the $m_{\pi}^{2}$ satisfies that

$$
\Pi_{\pi}\left(P^{2}=-m_{\pi}^{2}\right)=\Pi_{\pi}(0)-m_{\pi}^{2} N_{\pi}^{2}=0 .
$$

Therefore,

$$
\begin{aligned}
m_{\pi}^{2} N_{\pi}^{2} & =\Pi_{\pi}(P=0) \\
& =8 N_{c} \int \frac{\mathrm{d}^{4} p}{(2 \pi)^{4}} B_{m_{0}=0}\left(p^{2}\right)\left[\sigma_{B}^{m_{0}=0}-\frac{B_{m_{0}=0}}{B_{m_{0} \neq 0}} \sigma_{B}^{m_{0} \neq 0}\right] .
\end{aligned}
$$

This is just the formula we used in Eq. (11).
[1] C. S. Fischer, J. Luecker, and J. A. Mueller, Phys. Lett. B 702, 438 (2011).

[2] M. Mitter, J. M. Pawlowski, and N. Strodthoff, Phys. Rev. D 91, 054035 (2015).

[3] J. Braun, L. Fister, J. M. Pawlowski, and F. Rennecke, Phys. Rev. D 94, 034016 (2016).

[4] M. A. Stephanov, Proc. Sci., LAT2006 (2006) 024 [arXiv: hep-lat/0701002].

[5] W. Yuan, H. Chen, and Y. X. Liu, Phys. Lett. B 637, 69 (2006).

[6] J. Braun, L. M. Haas, F. Marhauser, and J. M. Pawlowski, Phys. Rev. Lett. 106, 022002 (2011).

[7] S. X. Qin, L. Chang, H. Chen, Y. X. Liu, and C. D. Roberts, Phys. Rev. Lett. 106, 172301 (2011).

[8] C. S. Fischer, J. Luecker, and C. A. Welzbacher, Phys. Rev. D 90, 034022 (2014).

[9] F. Gao and Y. X. Liu, Phys. Rev. D 94, 094030 (2016).

[10] J. N. Guenther, S. Borsanyi, Z. Fodor, S. K. Katz, K. K. Szab, A. Pasztor, I. Portillo, and C. Ratti, J. Phys. Conf. Ser. 1070, 012002 (2018).

[11] H. T. Ding et al., Phys. Rev. Lett. 123, 062002 (2019).

[12] C. S. Fischer, Prog. Part. Nucl. Phys. 105, 1 (2019).

[13] A. Bazavov et al. (HotQCD Collaboration), Phys. Lett. B 795, 15 (2019).

[14] W. J. Fu, J. M. Pawlowski, and F. Rennecke, Phys. Rev. D 101, 054032 (2020).

[15] F. Gao and J. M. Pawlowski, Phys. Rev. D 102, 034027 (2020)

[16] X. F. Luo and N. Xu, Nucl. Sci. Tech. 28, 112 (2017).

[17] L. Adamczyk et al., Phys. Rev. C 96, 044904 (2017).

[18] A. Andronic, P. Braun-Munzinger, K. Redlich, and J. Stachel, Nature (London) 561, 321 (2018).

[19] J. O. Andersen, W. R. Naylor, and A. Tranberg, Rev. Mod. Phys. 88, 025001 (2016).

[20] E. Shuryak, Rev. Mod. Phys. 89, 035001 (2017).

[21] J. M. Pawlowski, Nucl. Phys. A931, 113 (2014).

[22] Y. Yin, arXiv:1811.06519.

[23] C. D. Roberts and S. M. Schmidt, Prog. Part. Nucl. Phys. 45, S1 (2000).

[24] F. Wilczek, arXiv:hep-ph/003183.

[25] R. D. Pisarski and F. Wilczek, Phys. Rev. D 29, 338 (1984).

[26] M. Grahl and D. H. Rischke, Phys. Rev. D 88, 056014 (2013).

[27] T. Sato and N. Yamada, Phys. Rev. D 91, 034025 (2015).

[28] F. Gao, J. Chen, Y. X. Liu, S. X. Qin, C. D. Roberts, and S. M. Schmidt, Phys. Rev. D 93, 094019 (2016).
[29] J. Braun, W. J. Fu, J. M. Pawlowski, F. Rennecke, D. Rosenblüh, and S. Yin, Phys. Rev. D 102, 056010 (2020).

[30] D. Blaschke, A. Höll, C. D. Roberts, and S. Schmidt, Phys. Rev. C 58, 1758 (1998).

[31] A. Höll, P. Maris, and C. D. Roberts, Phys. Rev. C 59, 1751 (1999); P. Maris, arXiv:nucl-th/9908069.

[32] M. Blank and A. Krassnigg, Phys. Rev. D 82, 034006 (2010).

[33] C. S. Fischer and J. A. Mueller, Phys. Rev. D 84, 054013 (2011).

[34] K. Fukushima, K. Ohnishi, and K. Ohta, Phys. Rev. C 63, 045203 (2001).

[35] M. I. Buchoff et al. (LLNL/RBC Collaboration), Phys. Rev. D 89, 054514 (2014).

[36] T. Bhattacharya, M. I. Buchoff, N. H. Christ, H. T. Ding, R. Gupta, C. Jung et al. (HotQCD Collaboration), Phys. Rev. Lett. 113, 082001 (2014).

[37] V. Dick, F. Karsch, E. Laermann, S. Mukherjee, and S. Sharma, Phys. Rev. D 91, 094504 (2015).

[38] X. Li, W. J. Fu, and Y. X. Liu, Phys. Rev. D 101, 054034 (2020).

[39] Y. Hatta and T. Ikeda, Phys. Rev. D 67, 014028 (2003).

[40] F. Gao and Y. X. Liu, Phys. Rev. D 94, 076009 (2016).

[41] C. R. Allton, S. Ejiri, S. J. Hands, O. Kaczmarek, F. Karsch, E. Laermann, C. Schmidt, and L. Scorzato, Phys. Rev. D 66, 074507 (2002).

[42] R. V. Gavai and S. Gupta, Phys. Rev. D 68, 034506 (2003).

[43] C. R. Allton, S. Ejiri, S. J. Hands, O. Kaczmarek, F. Karsch, E. Laermann, and C. Schmidt, Phys. Rev. D 68, 014507 (2003).

[44] O. Kaczmarek, F. Karsch, E. Laermann, C. Miao, S. Mukherjee, P. Petreczky, C. Schmidt, W. Soeldner, and W. Unger, Phys. Rev. D 83, 014504 (2011).

[45] B. Klein, Phys. Rep. 707-708, 1 (2017).

[46] S. Resch, F. Rennecke, and B.-J. Schaefer, Phys. Rev. D 99, 076005 (2019).

[47] H. T. Ding, Nucl. Phys. A1005, 121940 (2021).

[48] N. Dupuis, L. Canet, A. Eichhorn, W. Metzner, J. Pawlowski, M. Tissier, and N. Wschebor, Phys. Rep. 910, 1 (2021).

[49] C. D. Roberts and A. G. Williams, Prog. Part. Nucl. Phys. 33, 477 (1994).

[50] P. Maris and C. D. Roberts, Int. J. Mod. Phys. E 12, 297 (2003); C. D. Roberts, Prog. Part. Nucl. Phys. 61, 50 (2008); I. C. Cloet and C. D. Roberts, Prog. Part. Nucl. Phys. 77, 1 (2014).

[51] R. Alkofer and L. von Smekal, Phys. Rep. 353, 281 (2001). 
[52] C. S. Fischer, J. Phys. G 32, R253 (2006).

[53] C. S. Fischer, A. Maas, and J. M. Pawlowski, Ann. Phys. (Amsterdam) 324, 2408 (2009).

[54] A. Bashir, L. Chang, I. C. Cloet, B. El-Bennich, Y. X. Liu, C. D. Roberts, and P. C. Tandy, Commun. Theor. Phys. 58, 79 (2012).

[55] S. X. Qin, L. Chang, Y. X. Liu, C. D. Roberts, and D. J. Wilson, Phys. Rev. C 84, 042202(R) (2011).

[56] H. J. Munczek and A. M. Nemirovsky, Phys. Rev. D 28, 181 (1983).

[57] M. R. Frank and C. D. Roberts, Phys. Rev. C 53, 390 (1996).

[58] A. Bender, G. I. Poulis, C. D. Roberts, S. Schmidt, and A. W. Thomas, Phys. Lett. B 431, 263 (1998).

[59] L. X. Gutiérrez-Guerrero, A. Bashir, I. C. Cloet, and C. D. Roberts, Phys. Rev. C 81, 065202 (2010).

[60] H. L. L. Roberts, A. Bashir, L. X. Gutierrez-Guerrero, C. D. Roberts, and D. J. Wilson, Phys. Rev. C 83, 065206 (2011).

[61] R. Contant and M. Q. Huber, Phys. Rev. D 96, 074002 (2017).

[62] H. J. Munczek, Phys. Rev. D 52, 4736 (1995).

[63] A. Bender, C. D. Roberts, and L. von Smekal, Phys. Lett. B 380, 7 (1996).

[64] P. Maris and C. D. Roberts, Phys. Rev. C 56, 3369 (1997).

[65] M. R. Frank and C. D. Roberts, Phys. Rev. C 53, 390 (1996).

[66] P. Maris and P. C. Tandy, Phys. Rev. C 60, 055214 (1999).

[67] E. Gutiérrez, A. Ahmad, A. Ayala, A. Bashir, and A. Raya, J. Phys. G 41, 075002 (2014).

[68] P. O. Bowman, U. M. Heller, D. B. Leinweber, M. B. Parappilly, and A. G. Williams, Phys. Rev. D 70, 034509 (2004).

[69] A. Sternbeck, E.-M. Ilgenfritz, M. Muller-Preussker, A. Schiller, and I. L. Bogolubsky, Proc. Sci., LAT2006 (2006) 076.

[70] A. Cucchieri and T. Mendes, Proc. Sci., LATTICE2007 (2007) 297.

[71] A. C. Aguilar, D. Binosi, and J. Papavassiliou, Phys. Rev. D 78, 025010 (2008).

[72] I. L. Bogolubsky, E.-M. Ilgenfritz, M. Müller-Preussker, and A. Sternbeck, Phys. Lett. B 676, 69 (2009).

[73] D. Zwanziger, Phys. Rev. D 87, 085039 (2013).

[74] A. K. Cyrol, M. Mitter, J. M. Pawlowski, and N. Strodthoff, Phys. Rev. D 97, 054006 (2018).
[75] M. Q. Huber, Phys. Rep. 879, 1 (2020).

[76] L. Chang, Y. X. Liu, C. D. Roberts, Y. M. Shi, W. M. Sun, and H. S. Zong, Phys. Rev. C 79, 035209 (2009).

[77] M. Y. Chen, M. H. Ding, L. Chang, and C. D. Roberts, Phys. Rev. D 98, 091505(R) (2018).

[78] P. Maris, C. D. Roberts, and S. M. Schmidt, Phys. Rev. C 57, R2821 (1998).

[79] M. Buballa, Phys. Rep. 407, 205 (2005).

[80] K. Raya, M. A. Bedolla, J. J. Cobos-Martínez, and A. Bashir, Few-Body Syst. 59, 133 (2018); L. X. GutiérrezGuerrero, A. Bashir, M. A. Bedolla, and E. Santopinto, Phy. Rev. D 100, 114032 (2019).

[81] P. L. Yin, C. Chen, G. Krein, C. D. Roberts, J. Segovia, and S. S. Xu, Phys. Rev. D 100, 034008 (2019).

[82] P. Maris, C. D. Roberts, and P. C. Tandy, Phys. Lett. B 420, 267 (1998).

[83] L. Chang, Y. X. Liu, M. S. Bhagwat, C. D. Roberts, and S. V. Wright, Phys. Rev. C 75, 015201 (2007).

[84] R. Williams, C. S. Fischer, and M. R. Pennington, Phys. Lett. B 645, 167 (2007).

[85] A. Bazavov, T. Bhattacharya, M. Cheng, C. DeTar, H. T. Ding, S. Gottlieb et al., Phys. Rev. D 85, 054503 (2012).

[86] C. McNeile, A. Bazavov, C. T. H. Davies, R. J. Dowdall, K. Hornbostel, G. P. Lepage, and H. D. Trottier, Phys. Rev. D 87, 034503 (2013).

[87] J. S. Ball and T. W. Chiu, Phys. Rev. D 22, 2542 (1980); 22, 2550 (1980).

[88] L. Chang, Y. X. Liu, and C. D. Roberts, Phys. Rev. Lett. 106, 072001 (2011).

[89] C. Tang, F. Gao, and Y. X. Liu, Phys. Rev. D 100, 056001 (2019).

[90] R. Williams, Eur. Phys. J. A 51, 57 (2015).

[91] R. Williams, C. S. Fischer, and W. Heupel, Phys. Rev. D 93, 034026 (2016).

[92] F. Gao, J. Papavassiliou, and J. M. Pawlowski, Phys. Rev. D 103, 094013 (2021).

[93] G. Eichmann, C. S. Fischer, and C. A. Welzbacher, Phys. Rev. D 93, 034013 (2016).

[94] R. Alkofer, C. S. Fischer, F. J. Llanes-Estrada, and K. Schwenzer, Ann. Phys. (Amsterdam) 324, 106 (2009).

[95] A. C. Aguilar, M. N. Ferreira, C. T. Figueiredo, and J. Papavassiliou, Phys. Rev. D 100, 094039 (2019).

[96] R. T. Cahill and C. D. Roberts, Phys. Rev. D 32, 2419 (1985). 